This document was prepared in conjunction with work accomplished under Contract No. DE-AC09-96SR18500 with the U. S. Department of Energy.

\title{
DISCLAIMER
}

This report was prepared as an account of work sponsored by an agency of the United States Government. Neither the United States Government nor any agency thereof, nor any of their employees, makes any warranty, express or implied, or assumes any legal liability or responsibility for the accuracy, completeness, or usefulness of any information, apparatus, product or process disclosed, or represents that its use would not infringe privately owned rights. Reference herein to any specific commercial product, process or service by trade name, trademark, manufacturer, or otherwise does not necessarily constitute or imply its endorsement, recommendation, or favoring by the United States Government or any agency thereof. The views and opinions of authors expressed herein do not necessarily state or reflect those of the United States Government or any agency thereof.

This report has been reproduced directly from the best available copy.

Available for sale to the public, in paper, from: U.S. Department of Commerce, National Technical Information Service, 5285 Port Royal Road, Springfield, VA 22161, phone: (800) 553-6847, fax: (703) 605-6900

email: orders@ntis.fedworld.gov

online ordering: http://www.ntis.gov/help/index.asp

Available electronically at http://www.osti.gov/bridge

Available for a processing fee to U.S. Department of Energy and its contractors, in paper, from: U.S. Department of Energy, Office of Scientific and Technical Information, P.O. Box 62, Oak Ridge, TN 37831-0062,

phone: (865)576-8401,

fax: (865)576-5728

email: $\underline{\text { reports@ adonis.osti.gov }}$ 
Keywords: Tank Farm

Saltstone

WAC

Tank $23 \mathrm{H}$ and $24 \mathrm{H}$

Characterization

\section{Retention Time:Permanent}

\section{Results of Analyses of Tank $23 \mathrm{H}$ and 24H Saltstone WAC Samples HTK-521 - HTK-528}

\section{R. F. Swingle, II}

Issue Date: March 12, 2003 


\section{SUMMARY}

Results of analyses of samples pulled from tanks $23 \mathrm{H}$ and $24 \mathrm{H}$ in November of 2002 are presented herein.

The initial samples pulled from the 60 -inch level of Tank $23 \mathrm{H}$ contained considerable solids. However, a later set of samples pulled from the 60 -inch level of Tank $23 \mathrm{H}$ indicated that the solids had settled. The initial Tank $23 \mathrm{H}$ composite sample was filtered. The filtrate and the solids were analyzed separately. The results of these analyses are documented herein.

\section{INTRODUCTION}

Eight samples were pulled from Tanks $23 \mathrm{H}$ and $24 \mathrm{H}$ in November of 2002 and transferred to the Savannah River Technology Center for analysis to determine whether material from the tanks could be transferred directly to the SPF for processing. The SPF, located in Z Area, is permitted as a wastewater treatment plant per the South Carolina Pollution Control Act, Title 48, Chapter 1. The aqueous waste is received and treated in the SPF to produce saltstone grout that is then transferred to the Saltstone Disposal Facility (SDF) for final disposal. The waste processed by the SPF must conform to requirements of this permit, additional Department of Energy (DOE) requirements and other limits imposed to protect the facility safety analysis. These limits are described in Procedure 4.01 of the Savannah River Site WAC Manual. The procedure also describes analytical checks required to verify compliance with the WAC prior to transferring aqueous waste to the Saltstone Production Facility. ${ }^{1}$ Aqueous waste meeting the WAC limits can be safely transferred, stored and treated in the SPF for subsequent disposal as saltstone in the SDF.

Because the schedule for this sample was considered critical, work on this sample was initiated prior to completion of the task technical and quality assurance plan with the approval of SRTC management. ${ }^{2}$ A task technical and quality assurance plan is currently being drafted for this work and will also serve as a guide for analysis of future Saltstone WAC samples.

\section{DISCUSSION}

\section{Experimental}

Two samples from each of the tanks at 2 different levels were pulled and composited. Table 1 shows how the samples were composited. Figure 1 shows the composited samples. All of the samples were relatively clear except the samples pulled from Tank $23 \mathrm{H}$ at the 60 -inch elevation. Each composite sample was divided and submitted to the SRTC Analytical Development Section (ADS) for the required analyses. 
Page 3 of 14

March 12, 2003

Table 1. Sampling Locations for Tanks $23 H$ and $24 \mathrm{H}$ Samples

\begin{tabular}{|c|c|c|c|}
\hline Composite Sample & Tank & Elevation above Tank Bottom & Sample IDs \\
\hline 1 & Tank 23 & 60 inches & HTK-521 \\
& & & HTK-522 \\
\hline 2 & Tank 23 & 100 inches & HTK-523 \\
& & & HTK-524 \\
\hline 3 & Tank 24H & 60 inches & HTK-525 \\
& & & HTK-527 \\
\hline 4 & Tank 24H & 100 inches & HTK-528 \\
\hline
\end{tabular}

Figure 1. Tanks 23H and 24H Samples

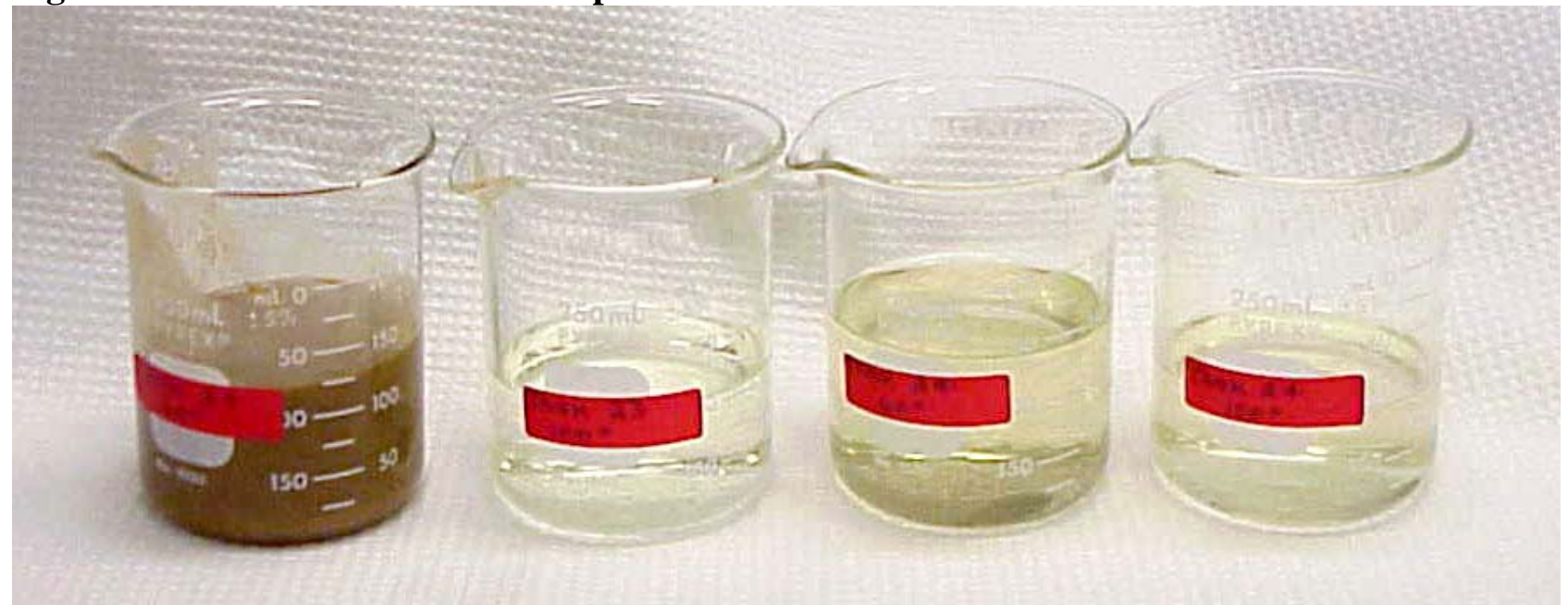

Left to Right: Tank 23H - 60 inches (HTK-521\&522), Tank 23H - 100 inches (HTK-523\&524), Tank 24H 60 inches (HTK-525\&526), Tank 24H - 100 inches (HTK-527\&528)

Two later samples were pulled from the 60" level of Tank 23H (HTK-529 and 530). The samples were relatively clear indicating the solids had settled (Figure 2). It was decided not to analyze the new samples since the original samples met with favorable results. 
Figure 2. Tank $23 \mathrm{H}$ - Second Set of Samples from 60"

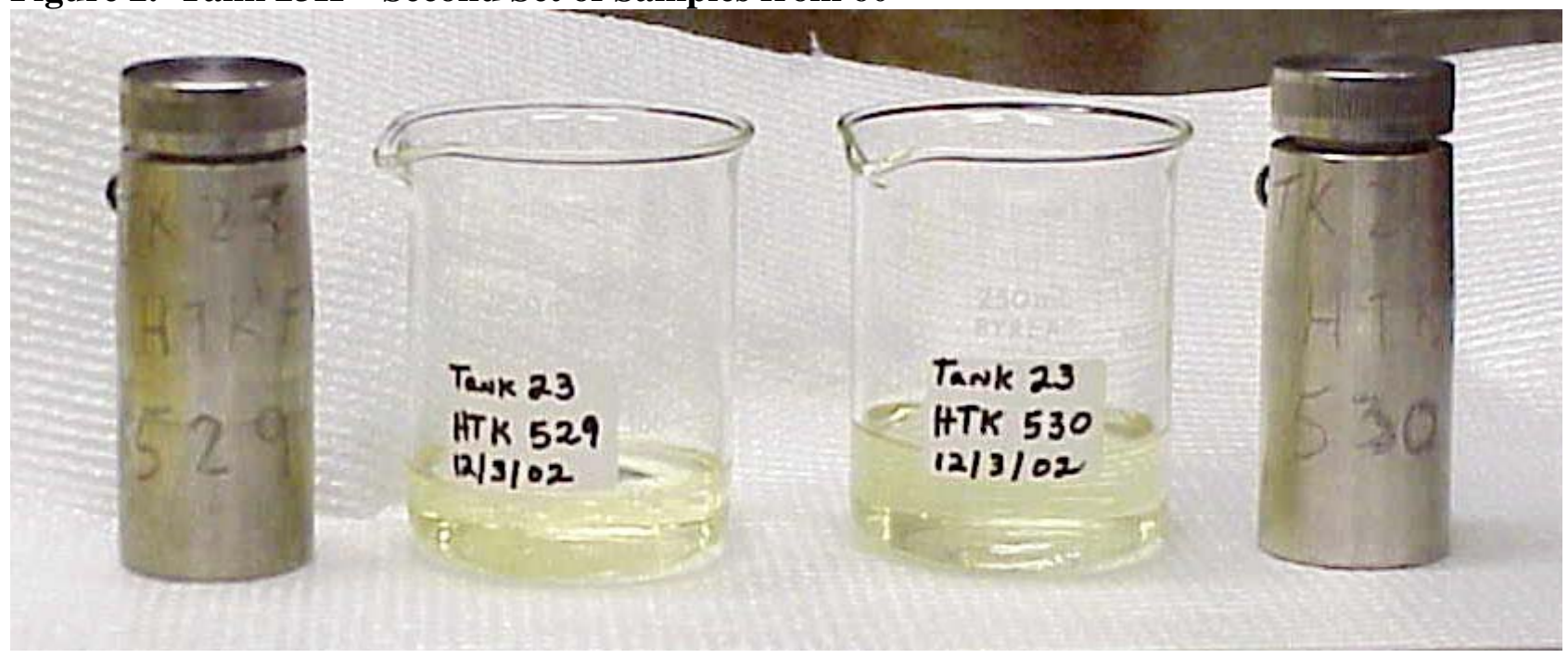

Supernate was analyzed for all four samples. The sample from the 60 -inch level of Tank $23 \mathrm{H}$ contained considerable solids. For this sample, both solids and filtrate were analyzed separately. Both values are given below.

Hazardous metals analysis was completed using inductively coupled plasma-emission spectroscopy (ICP-ES) for $\mathrm{Ag}, \mathrm{Ba}, \mathrm{Cd}, \mathrm{Cr}$ and $\mathrm{Pb}$ and atomic absorption (AA) for As and Se. Though not a part of the WAC, additional metals analyses were performed using ICP-ES and are included herein per request from the customer. Cold vapor atomic absorption (CVAA) was used to detect Hg. Gas chromatography/Mass spectrometry (GC/MS) was used to analyze for volatile and semivolatile organic compounds. Ion pair chromatography (IPC) was used to analyze for ethylenediaminetetraacetic acid (EDTA). No analysis was made for sodium tetraphenylborate (NaTPB) since process knowledge dictates that there is no NaTPB in the Tanks $23 \mathrm{H}$ or $24 \mathrm{H}$.

Radiochemical analyses were conducted using several counting methods. Chemical separations were performed prior to counting the samples for some of the species. The results are provided in units of $\mathrm{pCi} / \mathrm{mL}$.

Several different sample preparation methods were used, depending on the both the appearance of the sample and the analytical method employed. Sample preparation and analysis methods are given in Table 2. 
WSRC-TR-2003-00112

Revision 0

Page 5 of 14

March 12, 2003

Table 2. Sample Preparation and Analytical Methods

\begin{tabular}{|c|c|c|c|}
\hline Tank and Elevation & $\underline{\text { Sample Preparation }}$ & Analytical Methods & $\underline{\text { LIMS \#s }}$ \\
\hline \multirow[t]{7}{*}{ Tank $23 \mathrm{H}-60 "$} & None & $\begin{array}{c}\text { Volatile Organic } \\
\text { Semivolatile Organic } \\
\text { Ion-Pair Chromatography }\end{array}$ & 300189149 \\
\hline & None & $\begin{array}{c}\text { Free } \mathrm{OH} \\
\text { Carbonate } \\
\end{array}$ & 300189161 \\
\hline & $\begin{array}{l}\text { Filtration - Solids and } \\
\text { Acid Dissolution of } \\
\text { Solids }\end{array}$ & $\begin{array}{c}\text { ICP-ES, AA, CVAA, ICP-MS, } \\
\text { PuTTA }\end{array}$ & 300189175 \\
\hline & Filtration - Filtrate & $\begin{array}{c}\text { IC-anions, IC-Cations,ICP-ES, } \\
\text { AA, CVAA, ICP-MS }\end{array}$ & 300189177 \\
\hline & Filtration-Filtrate & $\begin{array}{c}\text { Pm-147/Sm-151, Pu-238/241. } \\
\text { Gamma Scan, Tk50 Rad } \\
\text { Screen, C-14, Ni-59/63, Sr-90, } \\
\text { Tc-99, I-129, tritium, Specific } \\
\text { Gravity }\end{array}$ & 300189178 \\
\hline & $\begin{array}{l}\text { Filtration-Solids and } \\
\text { Acid Dissolution of } \\
\text { Solids }\end{array}$ & $\begin{array}{l}\text { Pm-147/Sm-151, Pu-238/241. } \\
\text { Gamma Scan, Tk50 Rad } \\
\text { Screen, C-14, Ni-59/63, Sr-90, } \\
\text { Tc-99, I-129, tritium }\end{array}$ & 300189209 \\
\hline & None & $\begin{array}{c}\text { Suspended Solids, Dissolved } \\
\text { Solids, Particle Size }\end{array}$ & 300189179 \\
\hline \multirow[t]{6}{*}{$\begin{array}{l}\text { Tank } 23 \mathrm{H}-100 " \\
\text { Tank 24H - 60" } \\
\text { Tank 24H - 100” }\end{array}$} & None & $\begin{array}{c}\text { Volatile Organic } \\
\text { Semivolatile Organic } \\
\text { Ion-Pair Chromatography }\end{array}$ & $\begin{array}{l}300189149 \\
300189151 \\
300189152 \\
\end{array}$ \\
\hline & None & $\begin{array}{c}\text { Free } \mathrm{OH} \\
\text { Carbonate }\end{array}$ & $\begin{array}{l}300189159 \\
300189160 \\
300189158 \\
\end{array}$ \\
\hline & None & Soluble Solids & $\begin{array}{l}300189168 \\
300189169 \\
300189170 \\
\end{array}$ \\
\hline & None & $\begin{array}{l}\text { C-14, I-129, tritium, specific } \\
\text { gravity }\end{array}$ & $\begin{array}{l}300189171 \\
300189172 \\
300189167 \\
\end{array}$ \\
\hline & Acid Dissolution & $\begin{array}{c}\text { Ni-59/63, Sr-90. Tc-99. Pm- } \\
\text { 147/Sm-151, Pu-238/241, } \\
\text { Gamma Scan, Tk 50 Rad } \\
\text { Screen, ICP-ES, AA, CVAA, } \\
\text { ICP-MS }\end{array}$ & $\begin{array}{l}300189173 \\
300189174 \\
300189165 \\
300189166 \\
300189167 \\
\end{array}$ \\
\hline & None & IC-Cations, IC-Anions & $\begin{array}{l}300189164 \\
300189162 \\
300189163\end{array}$ \\
\hline
\end{tabular}




\section{Results}

Results for the Tank $23 \mathrm{H}$ samples are given in Table 3 but only the filtrate values have been included in the average for the tank. Results of the Tank 24H samples are given in Table 4. Results of each of two analyses are given along with an average and standard deviation. In cases where one or both of the results are below the limit of detection, no standard deviation is given. No attempt has been made to compare the results to the WAC limits, since at the time of preparation of this report, the WAC was being revised.

It should be noted that the filtration step could have removed solids that would not have settled by themselves. Therefore the Tank $23 \mathrm{H}-60$ " filtrate analyses may not be completely representative of material that would be transferred to Tank $50 \mathrm{H}$.

Many of the radionuclides requested are not expected to be present in measurable quantities in SRS waste. Some of these radionuclides are both time-consuming and expensive to analyze. For this reason the concentrations of many radionuclides are inferred rather than directly analyzed. For instance, we obtained a total alpha concentration. By inference, the concentration of individual alpha emitters cannot be any greater than the total alpha concentration. Therefore the total alpha also represents an upper limit on individual isotopes. Due to the statistics of counting, it is possible for an individually measured alpha-emitting isotope (e.g. ${ }^{238} \mathrm{Pu}$ ) to be measured at a slightly higher concentration than the total alpha concentration. The value given for $\mathrm{Pu}-239$ is actually a combination of $\mathrm{Pu}-239$ and $\mathrm{Pu}-240$.

For minor beta-gamma emitters, a similar method was employed. A gamma scan and total beta count were performed. This gave gross beta and some individual beta-gamma isotopes. A separate total beta count was performed after a chemical separation technique was used to remove the ${ }^{137} \mathrm{Cs}$. The ${ }^{90} \mathrm{Sr}$ and ${ }^{90} \mathrm{Y}$ results (twice the ${ }^{90} \mathrm{Sr}$ ) are subtracted from the Cs-free beta-gamma result to give a beta-gamma result that approximates the regulatory "other beta-gamma". Both values are given in the tables. Due to the statistics involved in counting, this calculated "other beta-gamma" result was negative for the Tank $24 \mathrm{H}$ samples. Since it appears that Tank 24 material will not be immediately transferred to Tank $50 \mathrm{H}$ for processing through the SPF, it was decided to report the these values as not detected (nd) in Table 4.

Ion-Chromatography analysis for the Tank $23 \mathrm{H}-60$ " sample was initially performed on an acidified sample aliquot, which affected both the nitrate and nitrite concentrations. Since a second composite pulled from the same location (HTK-529 and HTK-530) was available, it was submitted for IC. The IC results reported in Table 3 are from the HTK-529 and HTK-530 composite. All other results reported in the table are from the original composite (HTK-521 and HTK-522).

Table 3. Analytical Results of the Samples Pulled from Tank 23H

\begin{tabular}{|c|c|c|c|c|c|c|c|}
\hline \multirow{2}{*}{ Species } & \multicolumn{5}{|c|}{ Supernate } & \multicolumn{2}{|c|}{ Solids } \\
\hline & Units & 60" - Filtrate & $100^{\prime \prime}$ & Average & St. Dev. & Units & 60 " Solids \\
\hline \multicolumn{8}{|c|}{ Radiochemistry } \\
\hline \multicolumn{8}{|c|}{ Pu-238/241 } \\
\hline $\mathrm{Pu}-238$ & $\mathrm{pCi} / \mathrm{mL}$ & $4.00 \mathrm{E}+00$ & $2.51 \mathrm{E}+01$ & $1.45 \mathrm{E}+01$ & $1.49 \mathrm{E}+01$ & $\mathrm{dpm} / \mathrm{g}$ & $4.01 \mathrm{E}+06$ \\
\hline $\mathrm{Pu}-239$ & $\mathrm{pCi} / \mathrm{mL}$ & $<9.05 \mathrm{E}-01$ & $5.13 \mathrm{E}-01$ & $\leq 7.09 \mathrm{E}-01$ & - & $\mathrm{dpm} / \mathrm{g}$ & $<4.07 \mathrm{E}+04$ \\
\hline $\mathrm{Pu}-241$ & $\mathrm{pCi} / \mathrm{mL}$ & $<3.62 \mathrm{E}+01$ & $<3.81 \mathrm{E}+01$ & $<3.71 \mathrm{E}+01$ & - & $\mathrm{dpm} / \mathrm{g}$ & $6.48 \mathrm{E}+04$ \\
\hline \multicolumn{8}{|c|}{$\mathrm{Ni}-59 / 63$} \\
\hline $\mathrm{Ni}-59$ & $\mathrm{pCi} / \mathrm{mL}$ & $<1.00 \mathrm{E}+00$ & $<1.18 \mathrm{E}+00$ & $<1.09 \mathrm{E}+00$ & - & - & na \\
\hline $\mathrm{Ni}-63$ & $\mathrm{pCi} / \mathrm{mL}$ & $<9.19 \mathrm{E}+00$ & $<6.64 \mathrm{E}+00$ & $<7.92 \mathrm{E}+00$ & - & - & na \\
\hline
\end{tabular}


WSRC-TR-2003-00112

Revision 0

Page 7 of 14

March 12, 2003

Table 3 (cont'd). Analytical Results of the Samples Pulled from Tank 23H

\begin{tabular}{|c|c|c|c|c|c|c|c|}
\hline \multirow{2}{*}{ Species } & \multirow{2}{*}{ Units } & \multicolumn{4}{|c|}{ Supernate } & \multicolumn{2}{|c|}{ Solids } \\
\hline & & 60" - Filtrate & $100^{\prime \prime}$ & Average & St. Dev. & Units & 60 " Solids \\
\hline \multicolumn{8}{|l|}{ Tc-99 } \\
\hline Tc-99 & $\mathrm{pCi} / \mathrm{mL}$ & $2.17 \mathrm{E}+02$ & $1.38 \mathrm{E}+02$ & $1.78 \mathrm{E}+02$ & $5.55 \mathrm{E}+01$ & $\mathrm{dpm} / \mathrm{g}$ & $2.21 \mathrm{E}+03$ \\
\hline \multicolumn{8}{|l|}{ Sr-90 } \\
\hline Sr-90 & $\mathrm{pCi} / \mathrm{mL}$ & $<9.10 \mathrm{E}+01$ & $3.40 \mathrm{E}+02$ & $\leq 2.16 \mathrm{E}+02$ & - & $\mathrm{dpm} / \mathrm{g}$ & $1.28 \mathrm{E}+06$ \\
\hline \multicolumn{8}{|l|}{ Gamma Scan } \\
\hline Co-60 & $\mathrm{pCi} / \mathrm{mL}$ & $<5.72 \mathrm{E}+00$ & $<5.32 \mathrm{E}+00$ & $<5.52 \mathrm{E}+00$ & - & $\mathrm{dpm} / \mathrm{g}$ & $1.02 \mathrm{E}+04$ \\
\hline $\mathrm{Ru}-106$ & $\mathrm{pCi} / \mathrm{mL}$ & $<4.91 \mathrm{E}+01$ & $<4.48 \mathrm{E}+01$ & $<4.69 \mathrm{E}+01$ & - & $\mathrm{dpm} / \mathrm{g}$ & $<3.19 \mathrm{E}+03$ \\
\hline Sb-125 & $\mathrm{pCi} / \mathrm{mL}$ & $<1.67 \mathrm{E}+01$ & $<1.34 \mathrm{E}+01$ & $<1.50 \mathrm{E}+01$ & - & $\mathrm{dpm} / \mathrm{g}$ & $<1.80 \mathrm{E}+03$ \\
\hline Cs-137 & $\mathrm{pCi} / \mathrm{mL}$ & $8.15 E+04$ & $3.14 \mathrm{E}+04$ & $5.64 \mathrm{E}+04$ & $3.54 \mathrm{E}+04$ & $\mathrm{dpm} / \mathrm{g}$ & $5.07 \mathrm{E}+06$ \\
\hline Eu-154 & $\mathrm{pCi} / \mathrm{mL}$ & $<5.45 \mathrm{E}+00$ & $<5.14 \mathrm{E}+00$ & $<5.29 \mathrm{E}+00$ & - & $\mathrm{dpm} / \mathrm{g}$ & $2.16 \mathrm{E}+03$ \\
\hline Eu-155 & $\mathrm{pCi} / \mathrm{mL}$ & $<9.19 \mathrm{E}+00$ & $<8.20 \mathrm{E}+00$ & $<8.69 \mathrm{E}+00$ & - & $\mathrm{dpm} / \mathrm{g}$ & $<6.60 \mathrm{E}+02$ \\
\hline Al-26 & $\mathrm{pCi} / \mathrm{mL}$ & $<5.54 \mathrm{E}+00$ & $<4.68 \mathrm{E}+00$ & $<5.11 \mathrm{E}+00$ & - & - & na \\
\hline Nb-94 & $\mathrm{pCi} / \mathrm{mL}$ & $<4.91 \mathrm{E}+00$ & $<4.49 \mathrm{E}+00$ & $<4.70 \mathrm{E}+00$ & - & $\mathrm{dpm} / \mathrm{g}$ & $<8.44 \mathrm{E}+01$ \\
\hline Sn-126 & $\mathrm{pCi} / \mathrm{mL}$ & $<7.25 \mathrm{E}+00$ & $<6.80 \mathrm{E}+00$ & $<7.03 \mathrm{E}+00$ & - & $\mathrm{dpm} / \mathrm{g}$ & $<5.71 \mathrm{E}+02$ \\
\hline Eu-152 & $\mathrm{pCi} / \mathrm{mL}$ & $<3.11 \mathrm{E}+01$ & $<3.14 \mathrm{E}+01$ & $<3.13 \mathrm{E}+01$ & - & $\mathrm{dpm} / \mathrm{g}$ & $2.54 \mathrm{E}+03$ \\
\hline Ra-226 & $\mathrm{pCi} / \mathrm{mL}$ & $<9.82 \mathrm{E}+01$ & $<1.23 \mathrm{E}+02$ & $<1.11 \mathrm{E}+02$ & - & $\mathrm{dpm} / \mathrm{g}$ & $<7.65 \mathrm{E}+03$ \\
\hline $\mathrm{Pa}-231$ & $\mathrm{pCi} / \mathrm{mL}$ & $<1.34 \mathrm{E}+02$ & $<1.30 \mathrm{E}+02$ & $<1.32 \mathrm{E}+02$ & - & $\mathrm{dpm} / \mathrm{g}$ & $<1.47 \mathrm{E}+04$ \\
\hline Np-237 & $\mathrm{pCi} / \mathrm{mL}$ & $<9.37 \mathrm{E}+00$ & $<8.06 \mathrm{E}+00$ & $<8.72 \mathrm{E}+00$ & - & $\mathrm{dpm} / \mathrm{g}$ & $<1.01 \mathrm{E}+03$ \\
\hline Am-241 & $\mathrm{pCi} / \mathrm{mL}$ & $<1.67 \mathrm{E}+01$ & $<1.51 \mathrm{E}+01$ & $<1.59 \mathrm{E}+01$ & - & $\mathrm{dpm} / \mathrm{g}$ & $6.04 \mathrm{E}+04$ \\
\hline Ra-228 & $\mathrm{pCi} / \mathrm{mL}$ & $<2.45 \mathrm{E}+01$ & $<2.54 \mathrm{E}+01$ & $<2.50 \mathrm{E}+01$ & - & $\mathrm{dpm} / \mathrm{g}$ & $<6.18 \mathrm{E}+02$ \\
\hline Ac-227 & $\mathrm{pCi} / \mathrm{mL}$ & $<1.34 \mathrm{E}+02$ & $<1.30 \mathrm{E}+02$ & $<1.32 \mathrm{E}+02$ & - & - & na \\
\hline \multicolumn{8}{|c|}{ Derived from Other Beta-Gamma } \\
\hline Am-242m & $\mathrm{pCi} / \mathrm{mL}$ & $<9.58 \mathrm{E}+02$ & $<5.16 \mathrm{E}+02$ & $<7.38 \mathrm{E}+02$ & & - & na \\
\hline Bk-249 & $\mathrm{pCi} / \mathrm{mL}$ & $<7.92 \mathrm{E}+02$ & $<4.27 \mathrm{E}+02$ & $<6.10 \mathrm{E}+02$ & - & - & na \\
\hline \multicolumn{8}{|l|}{ Tritium } \\
\hline Tritium & $\mathrm{pCi} / \mathrm{mL}$ & $1.01 \mathrm{E}+03$ & $1.46 \mathrm{E}+03$ & $1.24 \mathrm{E}+03$ & $3.18 \mathrm{E}+02$ & $\mathrm{uCi} / \mathrm{g}$ & $1.02 \mathrm{E}-03$ \\
\hline \multicolumn{8}{|l|}{ Gross Alpha } \\
\hline Total Alpha & $\mathrm{pCi} / \mathrm{mL}$ & $<9.19 \mathrm{E}+01$ & $<8.75 \mathrm{E}+01$ & $<8.97 \mathrm{E}+01$ & - & $\mathrm{dpm} / \mathrm{g}$ & $3.90 \mathrm{E}+06$ \\
\hline \multicolumn{8}{|l|}{ Rad Screen } \\
\hline Total Beta & $\mathrm{pCi} / \mathrm{mL}$ & $9.78 \mathrm{E}+04$ & $3.77 \mathrm{E}+04$ & $6.77 \mathrm{E}+04$ & $4.25 \mathrm{E}+04$ & $\mathrm{dpm} / \mathrm{g}$ & $1.04 \mathrm{E}+07$ \\
\hline Beta/Gamma (Cs-Free) & $\mathrm{pCi} / \mathrm{mL}$ & $7.92 \mathrm{E}+02$ & $1.11 \mathrm{E}+03$ & $9.53 \mathrm{E}+02$ & $2.27 \mathrm{E}+02$ & - & na \\
\hline $\begin{array}{l}\text { Other Beta Gamma } \\
\text { (calculated) }\end{array}$ & $\mathrm{pCi} / \mathrm{mL}$ & $7.92 \mathrm{E}+02$ & $4.27 \mathrm{E}+02$ & $6.10 \mathrm{E}+02$ & $2.58 \mathrm{E}+02$ & - & na \\
\hline \multicolumn{8}{|l|}{ C-14 } \\
\hline C-14 & $\mathrm{pCi} / \mathrm{mL}$ & $3.01 \mathrm{E}+01$ & $1.87 \mathrm{E}+01$ & $2.44 \mathrm{E}+01$ & $8.09 \mathrm{E}+00$ & $\mathrm{dpm} / \mathrm{g}$ & $1.13 \mathrm{E}+03$ \\
\hline
\end{tabular}


WSRC-TR-2003-00112

Revision 0

Page 8 of 14

March 12, 2003

Table 3 (cont'd). Analytical Results of the Samples Pulled from Tank 23H

\begin{tabular}{|c|c|c|c|c|c|c|c|}
\hline \multirow{2}{*}{\begin{tabular}{|l|} 
Species \\
\end{tabular}} & \multicolumn{5}{|c|}{ Supernate } & \multicolumn{2}{|c|}{ Solids } \\
\hline & Units & 60" - Filtrate & $100 "$ & Average & St. Dev. & Units & 60" Solids \\
\hline \multicolumn{8}{|c|}{ Se-79 } \\
\hline Se-79 & $\mathrm{pCi} / \mathrm{mL}$ & - & - & $<7.33 \mathrm{E}+00$ & - & $\mathrm{dpm} / \mathrm{g}$ & $<1.04 \mathrm{E}+07$ \\
\hline \multicolumn{8}{|c|}{ I-129 } \\
\hline $\mathrm{I}-129$ & $\mathrm{pCi} / \mathrm{mL}$ & $<2.04 \mathrm{E}+00$ & $<6.58 \mathrm{E}-01$ & $<1.35 \mathrm{E}+00$ & - & $\mathrm{dpm} / \mathrm{g}$ & $3.88 \mathrm{E}+01$ \\
\hline \multicolumn{8}{|c|}{ Bound by Gross Alpha } \\
\hline $\mathrm{Cm}-248$ & $\mathrm{pCi} / \mathrm{mL}$ & $<1 \mathrm{E}+02$ & $<9.54 \mathrm{E}+01$ & $<9.78 \mathrm{E}+01$ & - & $\mathrm{dpm} / \mathrm{g}$ & $<4.25 \mathrm{E}+06$ \\
\hline Cf-252 & $\mathrm{pCi} / \mathrm{mL}$ & $<9.48 \mathrm{E}+01$ & $<9.03 \mathrm{E}+01$ & $<9.26 \mathrm{E}+01$ & - & $\mathrm{dpm} / \mathrm{g}$ & $<4.02 \mathrm{E}+06$ \\
\hline All other ${ }^{\alpha}$ & $\mathrm{pCi} / \mathrm{mL}$ & $<9.19 \mathrm{E}+01$ & $<8.75 \mathrm{E}+01$ & $<8.97 \mathrm{E}+01$ & - & $\mathrm{dpm} / \mathrm{g}$ & $<3.90 \mathrm{E}+06$ \\
\hline \multicolumn{8}{|c|}{ IPC } \\
\hline EDTA & $\mathrm{mg} / \mathrm{L}$ & $<2.00 \mathrm{E}+01$ & $<2.00 \mathrm{E}+01$ & $<2.00 \mathrm{E}+01$ & - & - & na \\
\hline \multicolumn{8}{|c|}{ ICP-ES } \\
\hline $\mathrm{Ag}$ & $\mathrm{mg} / \mathrm{L}$ & $<1.81 \mathrm{E}-02$ & $<9.00 \mathrm{E}-03$ & $<1.35 \mathrm{E}-02$ & - & ug/g & $6.72 \mathrm{E}-01$ \\
\hline $\mathrm{Al}$ & $\mathrm{mg} / \mathrm{L}$ & $2.23 \mathrm{E}+01$ & $5.56 \mathrm{E}+00$ & $1.39 \mathrm{E}+01$ & $1.18 \mathrm{E}+01$ & ug/g & $1.28 \mathrm{E}+02$ \\
\hline B & $\mathrm{mg} / \mathrm{L}$ & $4.74 \mathrm{E}+00$ & $3.74 \mathrm{E}-01$ & $2.56 \mathrm{E}+00$ & $3.09 \mathrm{E}+00$ & - & na \\
\hline $\mathrm{Ba}$ & $\mathrm{mg} / \mathrm{L}$ & $<4.22 \mathrm{E}-02$ & $<2.10 \mathrm{E}-02$ & $<3.16 \mathrm{E}-02$ & - & $\mathrm{ug} / \mathrm{g}$ & $4.82 \mathrm{E}+02$ \\
\hline $\mathrm{Be}$ & $\mathrm{mg} / \mathrm{L}$ & $<2.01 \mathrm{E}-03$ & $3.00 \mathrm{E}-03$ & $\leq 2.51 \mathrm{E}-03$ & - & $\mathrm{ug} / \mathrm{g}$ & $6.73 \mathrm{E}-01$ \\
\hline $\mathrm{Ca}$ & $\mathrm{mg} / \mathrm{L}$ & $5.10 \mathrm{E}+00$ & $3.17 \mathrm{E}+00$ & $4.13 \mathrm{E}+00$ & $1.36 \mathrm{E}+00$ & $\mathrm{ug} / \mathrm{g}$ & $1.05 \mathrm{E}+04$ \\
\hline $\mathrm{Cd}$ & $\mathrm{mg} / \mathrm{L}$ & $<9.05 \mathrm{E}-03$ & $<5.00 \mathrm{E}-03$ & $<7.02 \mathrm{E}-03$ & - & $\mathrm{ug} / \mathrm{g}$ & $1.30 \mathrm{E}+01$ \\
\hline $\mathrm{Cr}$ & $\mathrm{mg} / \mathrm{L}$ & $4.21 \mathrm{E}+00$ & $1.91 \mathrm{E}+00$ & $3.06 \mathrm{E}+00$ & $1.63 \mathrm{E}+00$ & $\mathrm{ug} / \mathrm{g}$ & $2.75 \mathrm{E}+02$ \\
\hline $\mathrm{Cu}$ & $\mathrm{mg} / \mathrm{L}$ & $8.94 \mathrm{E}-02$ & $8.20 \mathrm{E}-02$ & $8.57 \mathrm{E}-02$ & $5.26 \mathrm{E}-03$ & $\mathrm{ug} / \mathrm{g}$ & $1.05 \mathrm{E}+02$ \\
\hline $\mathrm{Fe}$ & $\mathrm{mg} / \mathrm{L}$ & $4.92 \mathrm{E}-02$ & $1.60 \mathrm{E}-01$ & $1.05 \mathrm{E}-01$ & $7.83 \mathrm{E}-02$ & $\mathrm{ug} / \mathrm{g}$ & $1.18 \mathrm{E}+04$ \\
\hline $\mathrm{Li}$ & $\mathrm{mg} / \mathrm{L}$ & $8.90 \mathrm{E}-01$ & $5.50 \mathrm{E}-02$ & $4.73 \mathrm{E}-01$ & $5.91 \mathrm{E}-01$ & $\mathrm{ug} / \mathrm{g}$ & $2.63 \mathrm{E}+01$ \\
\hline $\mathrm{Mg}$ & $\mathrm{mg} / \mathrm{L}$ & $8.44 \mathrm{E}-02$ & $<6.00 \mathrm{E}-03$ & $\leq 4.52 \mathrm{E}-02$ & - & $\mathrm{ug} / \mathrm{g}$ & $2.30 \mathrm{E}+03$ \\
\hline $\mathrm{Mn}$ & $\mathrm{mg} / \mathrm{L}$ & $<2.01 \mathrm{E}-03$ & $<1.00 \mathrm{E}-03$ & $<1.51 \mathrm{E}-03$ & - & $\mathrm{ug} / \mathrm{g}$ & $3.91 \mathrm{E}+02$ \\
\hline $\mathrm{Na}$ & $\mathrm{mg} / \mathrm{L}$ & $1.52 \mathrm{E}+04$ & $1.24 \mathrm{E}+04$ & $1.38 \mathrm{E}+04$ & $1.96 \mathrm{E}+03$ & ug/g & $2.88 \mathrm{E}+04$ \\
\hline $\mathrm{Ni}$ & $\mathrm{mg} / \mathrm{L}$ & 3.02E-02 & $1.50 \mathrm{E}-02$ & $2.26 \mathrm{E}-02$ & 1.07E-02 & $\mathrm{ug} / \mathrm{g}$ & $9.15 \mathrm{E}+01$ \\
\hline $\mathrm{P}$ & $\mathrm{mg} / \mathrm{L}$ & $4.48 \mathrm{E}+01$ & $3.26 \mathrm{E}+00$ & $2.40 \mathrm{E}+01$ & $2.94 \mathrm{E}+01$ & $\mathrm{ug} / \mathrm{g}$ & $3.42 \mathrm{E}+04$ \\
\hline$\overline{\mathrm{Pb}}$ & $\mathrm{mg} / \mathrm{L}$ & $1.17 \mathrm{E}-01$ & $<3.50 \mathrm{E}-02$ & $\leq 7.58 \mathrm{E}-02$ & - & $\mathrm{ug} / \mathrm{g}$ & $7.75 \mathrm{E}+02$ \\
\hline$S$ & $\mathrm{mg} / \mathrm{L}$ & $3.75 \mathrm{E}+01$ & $3.88 \mathrm{E}+01$ & $3.81 \mathrm{E}+01$ & $9.29 \mathrm{E}-01$ & $\mathrm{ug} / \mathrm{g}$ & $1.03 \mathrm{E}+03$ \\
\hline$\overline{\mathrm{Si}}$ & $\mathrm{mg} / \mathrm{L}$ & $2.61 \mathrm{E}+02$ & $1.09 \mathrm{E}+02$ & $1.85 \mathrm{E}+02$ & $1.08 \mathrm{E}+02$ & ug/g & $1.83 \mathrm{E}+05$ \\
\hline $\mathrm{Sr}$ & $\mathrm{mg} / \mathrm{L}$ & $1.09 \mathrm{E}+00$ & $6.89 \mathrm{E}-01$ & $8.87 \mathrm{E}-01$ & $2.80 \mathrm{E}-01$ & $\mathrm{ug} / \mathrm{g}$ & $2.40 \mathrm{E}+03$ \\
\hline $\mathrm{Ti}$ & $\mathrm{mg} / \mathrm{L}$ & $<1.71 \mathrm{E}-02$ & $<8.00 \mathrm{E}-03$ & $<1.25 \mathrm{E}-02$ & - & $\mathrm{ug} / \mathrm{g}$ & $2.81 \mathrm{E}+02$ \\
\hline $\mathrm{U}$ & $\mathrm{mg} / \mathrm{L}$ & $8.44 \mathrm{E}-01$ & $5.76 \mathrm{E}-01$ & $7.10 \mathrm{E}-01$ & $1.90 \mathrm{E}-01$ & $\mathrm{ug} / \mathrm{g}$ & $8.58 \mathrm{E}+02$ \\
\hline $\mathrm{V}$ & $\mathrm{mg} / \mathrm{L}$ & $7.54 \mathrm{E}-02$ & $3.20 \mathrm{E}-02$ & $5.37 \mathrm{E}-02$ & $3.07 \mathrm{E}-02$ & $\mathrm{ug} / \mathrm{g}$ & $1.48 \mathrm{E}+00$ \\
\hline $\mathrm{Zn}$ & $\mathrm{mg} / \mathrm{L}$ & $1.19 \mathrm{E}+00$ & $2.50 \mathrm{E}+00$ & $1.84 \mathrm{E}+00$ & $9.29 \mathrm{E}-01$ & $\mathrm{ug} / \mathrm{g}$ & $1.04 \mathrm{E}+03$ \\
\hline
\end{tabular}


Page 9 of 14

March 12, 2003

Table 3 (cont'd). Analytical Results of the Samples Pulled from Tank 23H

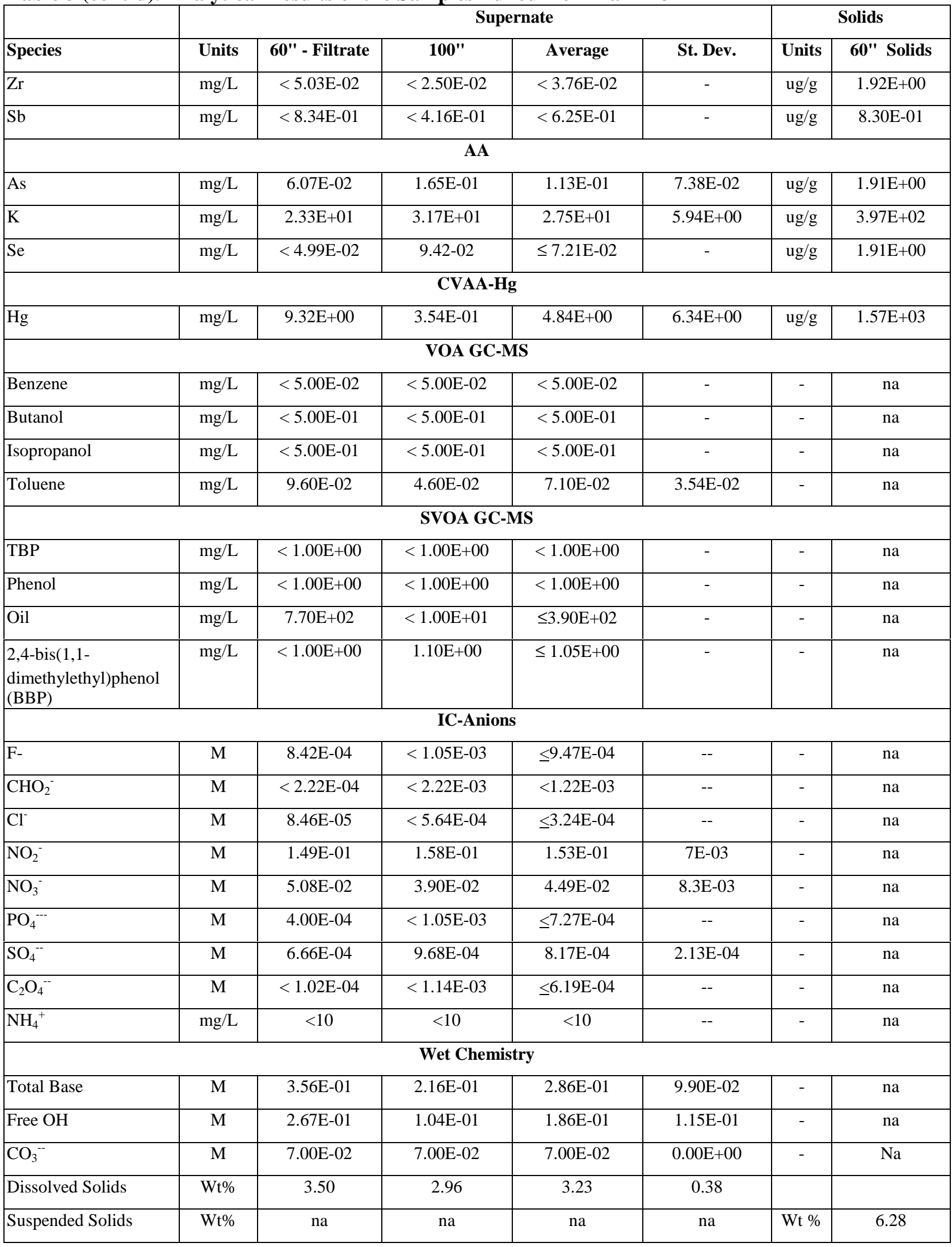




\section{March 12, 2003}

$\alpha$ All includes the following isotopes: Th-229, Th-230, Th-232, U-232, U-233, U-234, U-235, U-238, Pu-244, Am-243, $\mathrm{Cm}-242, \mathrm{Cm}-243, \mathrm{Cm}-245, \mathrm{Cm}-247$, Cf-249 and Cf-251. Since the gross alpha was below the detection limit, no single alpha isotope can be above the given lower limit of detection for total alpha. All of these isotopes decay $100 \%$ by alpha.

na Not analyzed.

Table4. Analytical Results of the Samples Pulled from Tank 24H

\begin{tabular}{|c|c|c|c|c|c|}
\hline \multirow{2}{*}{\begin{tabular}{|l|} 
\\
Species
\end{tabular}} & \multirow{2}{*}{\begin{tabular}{|l|} 
\\
Units \\
\end{tabular}} & \multicolumn{4}{|c|}{ Supernate } \\
\hline & & 60" & $100 "$ & Average & St. Dev. \\
\hline \multicolumn{6}{|c|}{ Radiochemistry } \\
\hline \multicolumn{6}{|c|}{ Pu-238/241 } \\
\hline $\mathrm{Pu}-238$ & $\mathrm{pCi} / \mathrm{mL}$ & $1.14 \mathrm{E}+03$ & $1.90 \mathrm{E}+02$ & $6.66 \mathrm{E}+02$ & $6.73 \mathrm{E}+02$ \\
\hline $\mathrm{Pu}-239$ & $\mathrm{pCi} / \mathrm{mL}$ & $9.92 \mathrm{E}+01$ & $1.55 \mathrm{E}+01$ & $5.74 \mathrm{E}+01$ & $5.92 \mathrm{E}+01$ \\
\hline $\mathrm{Pu}-241$ & $\mathrm{pCi} / \mathrm{mL}$ & $2.99 \mathrm{E}+02$ & $6.06 \mathrm{E}+01$ & $1.80 \mathrm{E}+02$ & $1.69 \mathrm{E}+02$ \\
\hline \multicolumn{6}{|c|}{$\mathrm{Ni}-59 / 63$} \\
\hline $\mathrm{Ni}-59$ & $\mathrm{pCi} / \mathrm{mL}$ & $<1.03 \mathrm{E}+00$ & $<3.78 \mathrm{E}-01$ & $<7.06 \mathrm{E}-01$ & - \\
\hline $\mathrm{Ni}-63$ & $\mathrm{pCi} / \mathrm{mL}$ & $<5.29 \mathrm{E}+01$ & $<2.74 \mathrm{E}+01$ & $<4.01 \mathrm{E}+01$ & - \\
\hline \multicolumn{6}{|c|}{ Tc-99 } \\
\hline Tc-99 & $\mathrm{pCi} / \mathrm{mL}$ & $2.60 \mathrm{E}+02$ & $3.99 \mathrm{E}+02$ & $3.29 \mathrm{E}+02$ & $9.84 \mathrm{E}+01$ \\
\hline \multicolumn{6}{|c|}{ Sr-90 } \\
\hline Sr-90 & $\mathrm{pCi} / \mathrm{mL}$ & $4.52 \mathrm{E}+04$ & $5.74 \mathrm{E}+04$ & $5.13 \mathrm{E}+04$ & $8.60 \mathrm{E}+03$ \\
\hline \multicolumn{6}{|c|}{ Gamma Scan } \\
\hline Co-60 & $\mathrm{pCi} / \mathrm{mL}$ & $<5.45 \mathrm{E}+00$ & $<5.59 \mathrm{E}+00$ & $<5.52 \mathrm{E}+00$ & - \\
\hline $\mathrm{Ru}-106$ & $\mathrm{pCi} / \mathrm{mL}$ & $<1.47 \mathrm{E}+02$ & $<9.50 \mathrm{E}+01$ & $<1.21 \mathrm{E}+02$ & - \\
\hline Sb-125 & $\mathrm{pCi} / \mathrm{mL}$ & $<7.07 \mathrm{E}+01$ & $<5.59 \mathrm{E}+00$ & $<3.82 \mathrm{E}+01$ & - \\
\hline Cs-137 & $\mathrm{pCi} / \mathrm{mL}$ & $4.02 \mathrm{E}+06$ & $1.51 \mathrm{E}+06$ & $2.77 \mathrm{E}+06$ & $1.78 \mathrm{E}+06$ \\
\hline Eu-154 & $\mathrm{pCi} / \mathrm{mL}$ & $<1.80 \mathrm{E}+01$ & $<1.52 \mathrm{E}+01$ & $<1.66 \mathrm{E}+01$ & - \\
\hline Eu-155 & $\mathrm{pCi} / \mathrm{mL}$ & $<3.18 \mathrm{E}+01$ & $<2.37 \mathrm{E}+01$ & $<2.78 \mathrm{E}+01$ & - \\
\hline Al-26 & $\mathrm{pCi} / \mathrm{mL}$ & $<5.23 \mathrm{E}+00$ & $<5.99 \mathrm{E}+00$ & $<5.61 \mathrm{E}+00$ & - \\
\hline $\mathrm{Nb}-94$ & $\mathrm{pCi} / \mathrm{mL}$ & $<5.05 \mathrm{E}+00$ & $<5.14 \mathrm{E}+00$ & $<5.09 \mathrm{E}+00$ & - \\
\hline Sn-126 & $\mathrm{pCi} / \mathrm{mL}$ & $<2.78 \mathrm{E}+01$ & $<2.06 \mathrm{E}+01$ & $<2.42 \mathrm{E}+01$ & - \\
\hline Eu-152 & $\mathrm{pCi} / \mathrm{mL}$ & $<3.89 \mathrm{E}+01$ & $<3.86 \mathrm{E}+01$ & $<3.87 \mathrm{E}+01$ & - \\
\hline Ra-226 & $\mathrm{pCi} / \mathrm{mL}$ & $<3.48 \mathrm{E}+02$ & $<2.32 \mathrm{E}+02$ & $<2.90 \mathrm{E}+02$ & - \\
\hline $\mathrm{Pa}-231$ & $\mathrm{pCi} / \mathrm{mL}$ & $<5.72 \mathrm{E}+02$ & $<3.56 \mathrm{E}+02$ & $<4.64 \mathrm{E}+02$ & - \\
\hline Np-237 & $\mathrm{pCi} / \mathrm{mL}$ & $<3.86 \mathrm{E}+01$ & $<2.41 \mathrm{E}+01$ & $<3.13 \mathrm{E}+01$ & - \\
\hline Am-241 & $\mathrm{pCi} / \mathrm{mL}$ & $<6.58 \mathrm{E}+01$ & $<5.05 \mathrm{E}+01$ & $<5.81 \mathrm{E}+01$ & - \\
\hline Ra-228 & $\mathrm{pCi} / \mathrm{mL}$ & $<2.58 \mathrm{E}+01$ & $<2.62 \mathrm{E}+01$ & $<2.60 \mathrm{E}+01$ & - \\
\hline Ac-227 & $\mathrm{pCi} / \mathrm{mL}$ & $<5.72 \mathrm{E}+02$ & $<3.56 \mathrm{E}+02$ & $<4.64 \mathrm{E}+02$ & - \\
\hline
\end{tabular}


WSRC-TR-2003-00112

Revision 0

Page 11 of 14

March 12, 2003

Table4 (cont'd). Analytical Results of the Samples Pulled from Tank 24H

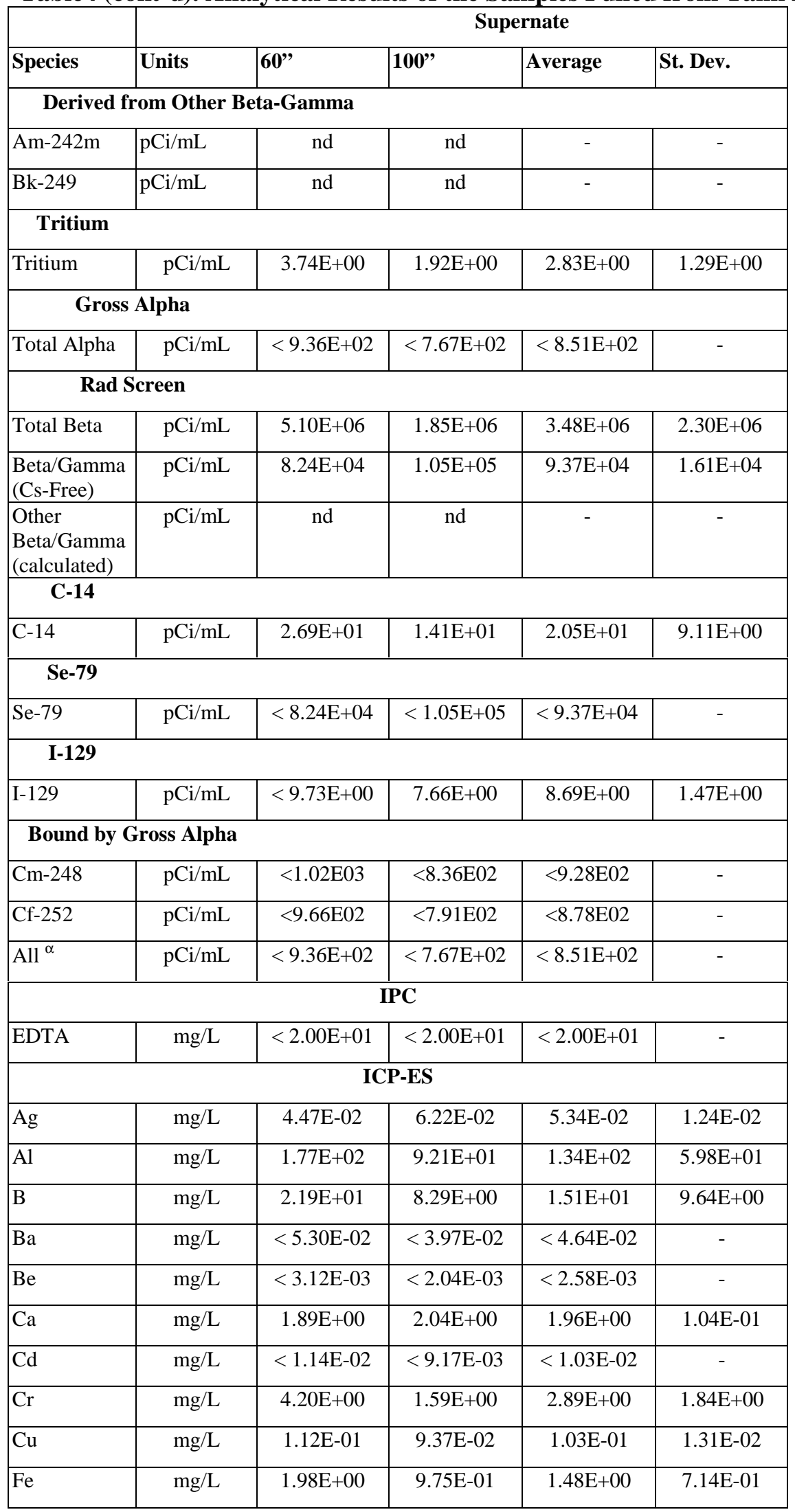


WSRC-TR-2003-00112

Revision 0

Page 12 of 14

March 12, 2003

Table4 (cont'd). Analytical Results of the Samples Pulled from Tank 24H

\begin{tabular}{|c|c|c|c|c|c|}
\hline \multirow[b]{2}{*}{ Species } & \multirow{2}{*}{ Units } & \multicolumn{4}{|c|}{ Supernate } \\
\hline & & 60"' & $100 "$ & Average & St. Dev. \\
\hline $\mathrm{Li}$ & $\mathrm{mg} / \mathrm{L}$ & $1.78 \mathrm{E}+01$ & $2.75 \mathrm{E}+00$ & $1.03 \mathrm{E}+01$ & $1.06 \mathrm{E}+01$ \\
\hline $\mathrm{Mg}$ & $\mathrm{mg} / \mathrm{L}$ & $4.57 \mathrm{E}-02$ & $1.63 \mathrm{E}-02$ & $3.10 \mathrm{E}-02$ & $2.08 \mathrm{E}-02$ \\
\hline $\mathrm{Mn}$ & $\mathrm{mg} / \mathrm{L}$ & $1.97 \mathrm{E}-02$ & $9.17 \mathrm{E}-03$ & $1.45 \mathrm{E}-02$ & 7.47E-03 \\
\hline $\mathrm{Na}$ & $\mathrm{mg} / \mathrm{L}$ & $2.92 \mathrm{E}+04$ & $2.35 \mathrm{E}+04$ & $2.64 \mathrm{E}+04$ & $4.00 \mathrm{E}+03$ \\
\hline $\mathrm{Ni}$ & $\mathrm{mg} / \mathrm{L}$ & $3.84 \mathrm{E}-02$ & $<2.85 \mathrm{E}-02$ & $\leq 3.35 \mathrm{E}-02$ & 7.01E-03 \\
\hline $\mathrm{P}$ & $\mathrm{mg} / \mathrm{L}$ & $1.64 \mathrm{E}+01$ & $6.84 \mathrm{E}+00$ & $1.16 \mathrm{E}+01$ & $6.77 \mathrm{E}+00$ \\
\hline $\mathrm{Pb}$ & $\mathrm{mg} / \mathrm{L}$ & $<8.94 \mathrm{E}-02$ & $<6.73 \mathrm{E}-02$ & $<7.83 \mathrm{E}-02$ & - \\
\hline$S$ & $\mathrm{mg} / \mathrm{L}$ & $4.52 \mathrm{E}+01$ & $2.01 \mathrm{E}+01$ & $3.26 \mathrm{E}+01$ & $1.78 \mathrm{E}+01$ \\
\hline $\mathrm{Si}$ & $\mathrm{mg} / \mathrm{L}$ & $6.51 \mathrm{E}+01$ & $4.74 \mathrm{E}+01$ & $5.63 \mathrm{E}+01$ & $1.26 \mathrm{E}+01$ \\
\hline $\mathrm{Sr}$ & $\mathrm{mg} / \mathrm{L}$ & $4.18 \mathrm{E}-01$ & $4.40 \mathrm{E}-01$ & $4.29 \mathrm{E}-01$ & $1.59 \mathrm{E}-02$ \\
\hline $\mathrm{Ti}$ & $\mathrm{mg} / \mathrm{L}$ & $2.18 \mathrm{E}-02$ & $1.63 \mathrm{E}-02$ & $1.91 \mathrm{E}-02$ & $3.90 \mathrm{E}-03$ \\
\hline $\mathrm{U}$ & $\mathrm{mg} / \mathrm{L}$ & $6.61 \mathrm{E}+00$ & $1.06 \mathrm{E}+01$ & $8.60 \mathrm{E}+00$ & $2.82 \mathrm{E}+00$ \\
\hline V & $\mathrm{mg} / \mathrm{L}$ & 2.29E-02 & $7.13 \mathrm{E}-03$ & $1.50 \mathrm{E}-02$ & $1.11 \mathrm{E}-02$ \\
\hline $\mathrm{Zn}$ & $\mathrm{mg} / \mathrm{L}$ & $4.11 \mathrm{E}-01$ & $4.35 \mathrm{E}-01$ & $4.23 \mathrm{E}-01$ & $1.67 \mathrm{E}-02$ \\
\hline $\mathrm{Zr}$ & $\mathrm{mg} / \mathrm{L}$ & $<6.34 \mathrm{E}-02$ & $<4.79 \mathrm{E}-02$ & $<5.56 \mathrm{E}-02$ & - \\
\hline $\mathrm{Sb}$ & $\mathrm{mg} / \mathrm{L}$ & $<1.06 \mathrm{E}+00$ & $<7.97 \mathrm{E}-01$ & $<9.28 \mathrm{E}-01$ & - \\
\hline \multicolumn{6}{|c|}{$\mathbf{A A}$} \\
\hline As & $\mathrm{mg} / \mathrm{L}$ & $3.08 \mathrm{E}-01$ & $4.83 \mathrm{E}-02$ & $1.78 \mathrm{E}-01$ & $1.84 \mathrm{E}-01$ \\
\hline $\mathrm{K}$ & $\mathrm{mg} / \mathrm{L}$ & $2.65 \mathrm{E}+01$ & $1.47 \mathrm{E}+01$ & $2.06 \mathrm{E}+01$ & $8.34 \mathrm{E}+00$ \\
\hline $\mathrm{Se}$ & $\mathrm{mg} / \mathrm{L}$ & $6.62 \mathrm{E}-02$ & $<4.70 \mathrm{E}-02$ & $\leq 5.66 \mathrm{E}-02$ & $1.36 \mathrm{E}-02$ \\
\hline \multicolumn{6}{|c|}{ CVAA-Hg } \\
\hline $\mathrm{Hg}$ & $\mathrm{mg} / \mathrm{L}$ & $1.35 \mathrm{E}+01$ & $1.45 \mathrm{E}+01$ & $1.40 \mathrm{E}+01$ & 7.07E-01 \\
\hline \multicolumn{6}{|c|}{ VOA GC-MS } \\
\hline Benzene & $\mathrm{mg} / \mathrm{L}$ & $<5.00 \mathrm{E}-02$ & $<5.00 \mathrm{E}-02$ & $<5.00 \mathrm{E}-02$ & - \\
\hline Butanol & $\mathrm{mg} / \mathrm{L}$ & $<5.00 \mathrm{E}-01$ & $<5.00 \mathrm{E}-01$ & $<5.00 \mathrm{E}-01$ & - \\
\hline Isopropanol & $\mathrm{mg} / \mathrm{L}$ & $<5.00 \mathrm{E}-01$ & $<5.00 \mathrm{E}-01$ & $<5.00 \mathrm{E}-01$ & - \\
\hline Toluene & $\mathrm{mg} / \mathrm{L}$ & $6.30 \mathrm{E}-02$ & $1.00 \mathrm{E}-01$ & $8.15 \mathrm{E}-02$ & $2.62 \mathrm{E}-02$ \\
\hline \multicolumn{6}{|c|}{ SVOA GC-MS } \\
\hline TBP & $\mathrm{mg} / \mathrm{L}$ & $<1.00 \mathrm{E}+00$ & $<1.00 \mathrm{E}+00$ & $<1.00 \mathrm{E}+00$ & - \\
\hline Phenol & $\mathrm{mg} / \mathrm{L}$ & $<1.00 \mathrm{E}+00$ & $<1.00 \mathrm{E}+00$ & $<1.00 \mathrm{E}+00$ & - \\
\hline Oil & $\mathrm{mg} / \mathrm{L}$ & $<2.00 \mathrm{E}+01$ & $<2.00 \mathrm{E}+01$ & $<2.00 \mathrm{E}+01$ & - \\
\hline BBP & $\mathrm{mg} / \mathrm{L}$ & $<1.00 \mathrm{E}+00$ & $1.20 \mathrm{E}+00$ & $1.10 \mathrm{E}+00$ & $1.41 \mathrm{E}-01$ \\
\hline \multicolumn{6}{|c|}{ IC-Anions } \\
\hline $\mathrm{F}^{-}$ & $\mathrm{M}$ & $<1.05 \mathrm{E}-03$ & $<1.05 \mathrm{E}-03$ & $<1.05 \mathrm{E}-03$ & - \\
\hline $\mathrm{CHO}_{2}^{-}$ & $\mathrm{M}$ & $1.67 \mathrm{E}-03$ & $2.75 \mathrm{E}-03$ & $2.21 \mathrm{E}-03$ & $7.70 \mathrm{E}-04$ \\
\hline
\end{tabular}


WSRC-TR-2003-00112

Revision 0

Page 13 of 14

March 12, 2003

Table4 (cont'd). Analytical Results of the Samples Pulled from Tank 24H

\begin{tabular}{|c|c|c|c|c|c|}
\hline & & \multicolumn{4}{|c|}{ Supernate } \\
\hline Species & Units & 60" & $100 "$ & Average & St. Dev. \\
\hline $\mathrm{Cl}^{-}$ & $\mathrm{M}$ & $2.51 \mathrm{E}-03$ & $9.59 \mathrm{E}-04$ & $1.73 \mathrm{E}-03$ & $1.10 \mathrm{E}-03$ \\
\hline $\mathrm{NO}_{2}^{-}$ & M & $4.20 \mathrm{E}-01$ & $3.15 \mathrm{E}-01$ & $3.67 \mathrm{E}-01$ & $7.38 \mathrm{E}-02$ \\
\hline $\mathrm{NO}_{3}^{-}$ & M & $3.42 \mathrm{E}-02$ & $6.14 \mathrm{E}-02$ & $4.78 \mathrm{E}-02$ & $1.93 \mathrm{E}-02$ \\
\hline $\mathrm{PO}_{4}^{---}$ & M & $<1.05 \mathrm{E}-03$ & $<1.05 \mathrm{E}-03$ & $<1.05 \mathrm{E}-03$ & - \\
\hline $\mathrm{SO}_{4}^{--}$ & $\mathrm{M}$ & $7.70 \mathrm{E}-04$ & $4.06 \mathrm{E}-04$ & $5.88 \mathrm{E}-04$ & $2.58 \mathrm{E}-04$ \\
\hline $\mathrm{C}_{2} \mathrm{O}_{4}^{--}$ & M & $<1.14 \mathrm{E}-03$ & $<1.14 \mathrm{E}-03$ & $<1.14 \mathrm{E}-03$ & - \\
\hline $\mathrm{NH}_{4}^{+}$ & $\mathrm{mg} / \mathrm{L}$ & $<10$ & $<10$ & $<10$ & -- \\
\hline \multicolumn{6}{|c|}{ Wet Chemistry } \\
\hline Total Base & M & $5.16 \mathrm{E}-01$ & $5.58 \mathrm{E}-01$ & 5.37E-01 & $2.97 \mathrm{E}-02$ \\
\hline Free $\mathrm{OH}$ & M & $2.85 \mathrm{E}-01$ & $4.35 \mathrm{E}-01$ & $3.60 \mathrm{E}-01$ & $1.06 \mathrm{E}-01$ \\
\hline $\mathrm{CO}_{3}^{--}$ & $\mathrm{M}$ & $1.50 \mathrm{E}-01$ & $7.00 \mathrm{E}-02$ & $1.10 \mathrm{E}-01$ & $5.66 \mathrm{E}-02$ \\
\hline $\begin{array}{l}\text { Dissolved } \\
\text { Solids }\end{array}$ & $\mathrm{Wt} \%$ & 6.93 & 5.67 & 6.30 & 0.89 \\
\hline
\end{tabular}

$\alpha$ All includes the following isotopes: Th-229, Th-230, Th-232, U-232, U-233, U-234, U-235, U-238, Pu244, Am-243, Cm-242, Cm-243, Cm-245, Cm-247, Cf-249 and Cf-251. Since no alpha was detected, no single alpha isotope can be above the given lower limit of detection for total alpha. All of these isotopes decay $100 \%$ by alpha

nd Other beta-gamma was calculated by subtracting the Sr-90, Y-90 and Tc-99 from the Cs-free total beta. The result was a negative value indicating that within the counting statistics Sr-90, Y-90 and Tc-99 acoiunt for all of the Cs-free total beta. Because a negative number is not physically possible, this is reported as not detected or "nd".

\section{Quality Assurance}

Data for these analyses are recorded in laboratory notebooks WSRC-NB-2003-00024 and succeeding notebooks.

\section{Acknowledgements}

The author gratefully acknowledges the assistance of A. L. Williams of WPT, D. M. Ferrara, D. P. Diprete, C. J. Coleman, R. J. Ray, J. C. Hart, A. A. Ekechukwu and L. W. Brown of ADS in the completion of this work.

\section{References}

1 "Acceptance Criteria for Aqueous Waste Sent to the Z Area Saltstone Production Facility," Manual 1S, Procedure WAC 4.01, Revision 3, February 25, 2002.

${ }^{2}$ W. B. Van Pelt, "Exemption to Start Work - Characterization of Tanks $23 \mathrm{H}$ and $24 \mathrm{H}$ Samples with Parallel Development of a Technical Task Plan," SRT-LWP-2002-00131, November 22, 2002. 
Page 14 of 14

March 12, 2003 